\title{
Evaluation de la valeur économique directe de l'élevage bovin au nord-Bénin
}

\author{
K. L. S. Adam SOUNON ${ }^{1 *}$, Edouard AHO ${ }^{2}$, P. LESSE ${ }^{3}$, Alexandre ICKOWICZ ${ }^{4,5}$, \\ Samir MESSAD ${ }^{4,5}$, Matthieu LESNOFF ${ }^{4,5}$ et Marcel R. B. HOUINATO ${ }^{3}$ \\ ${ }^{1}$ Institut National des Recherches Agricoles du Bénin (INRAB), Abomey-Calavi, Bénin. \\ ${ }^{2}$ Ecole Nationale d'Administration et de Magistrature (ENAM), Abomey-Calavi, Bénin. \\ ${ }^{3}$ Faculté des Sciences Agronomiques de l'Université d'Abomey-Calavi, Abomey-Calavi, Bénin. \\ ${ }^{4}$ CIRAD, UMR SELMET, F-34398 Montpellier, France. \\ ${ }^{5}$ SELMET, Univ Montpellier, CIRAD, INRA, Montpellier SupAgro, Montpellier, France. \\ *Auteur correspondant ; E-mail : kone907@yahoo.fr; Tél. : 00 (229) 96000388 ;
}

\section{RESUME}

Dans l'espace ouest-africain, la demande en produits d'origine animale augmente au rythme de la croissance de la population, de l'urbanisation et de l'accroissement des revenus. Cette situation offre un marché potentiel de bétail, de viande et de lait. Mais le paradoxe demeure la part de plus en plus croissante des importations extra africaines de produits animaux. L'économie pastorale éprouve des difficultés réelles à faire face à l'accroissement de la demande de viande et de lait dans les pays ouest-africains. C'est pourquoi l'un des défis majeurs auxquels la filière bétail /viande et lait est confronté est d'assurer aux populations un niveau suffisant de consommation de viande et de lait tout en développant les exportations. Cependant, la Revue Economique Mondiale du Pastoralisme a illustré dans ces travaux l'état de pauvreté du savoir sur les économies pastorales, le manque de collecte de données dans certains pays et l'absence de désagrégation des données nationales dans d'autres pays. C'est pour contribuer à combler le déficit de savoirs sur les économies pastorales que la présente étude a été consacrée à l'évaluation de la valeur économique de l'élevage bovin au Bénin à partir d'un bassin de production. Elle permettra de fournir des chiffres capables de générer des critères mieux avisés pour permettre l'investissement public et la prise de décisions politiques en faveur de l'élevage bovin. Cette étude s'est appuyée sur des enquêtes menées au niveau de 52 exploitations d'élevages bovin de deux principaux systèmes naisseurs, choisis de manière raisonnée dans 12 localités représentatives de la diversité du bassin de la Donga. La démarche méthodologique suivie pour évaluer la valeur économique des productions bovine, se décline en trois étapes : la recension documentaire, l'estimation des productions et leur conversion en valeur monétaire. Au terme de l'évaluation, la valeur économique totale de la production bovine dans le bassin de la Donga, représentée par les productions d'animaux sur pied, de viande et de lait, est de un milliard neuf millions quatre cent mille (1 009400 000) francs CFA. Rapportée à la population du bassin, cette valeur économique de la production bovine est de 4143 FCFA/habitant. Comparée à la valeur totale de production d'aliments en 2012 au Bénin qui est de 99000 FCFA/habitant, la valeur de la production bovine représente $4 \%$. Ce résultat met en évidence la faible participation de l'élevage bovin dans la production de l'aliment au Bénin.

(C) 2019 International Formulae Group. All rights reserved.

Mots clés : Valeur économique, systèmes d'élevage, bovin, produits animaux, Bénin. 


\title{
Assessment of the direct economic value of cattle herding in northern Benin
}

\begin{abstract}
In the West African area, demand for animal products is growing in line with population growth, urbanization and rising incomes. This situation offers a potential market of meat and milk for livestock. But the paradox remains the growing importation rate of animal products from extra-African. The economy of pastoral activities is facing real difficulties in coping with the increasing demand for meat and milk in West African countries. This is why one of the major challenges in meat and milk sector is to ensure that people have a sufficient level of consumption of meat and milk while developing exportation. However, the World Economic Review of Pastoralism illustrated in this work the state of poverty of knowledge about pastoral economies, the lack of data collection in some countries and the lack of disaggregation of national data in other countries. It is to help fill the knowledge gap on pastoral economies that this study has been devoted to assessing the economic value of cattle farming in Benin from a production base. It will provide figures capable of generating better criteria for public investment and political decision-making in favor of cattle breeding. This study was based on surveys conducted at 52 cattle farms of two main systems, carefully selected in 12 localities representative of the diversity of the Donga district. The methodological approach followed to evaluate the economic value of cattle production, is divided into three stages: literature review, the estimation of the productions and their conversion into monetary value. At the end of the appraisal, the total economic value of beef production in the Donga Basin, represented by the production of live animals, meat and milk, is one billion nine million four hundred thousand $(1,009,400,000)$ CFA francs. Relative to the population of the area, this economic value of the cattle production is 4143 FCFA / inhabitant. Compared with the total value of food production in Benin in 2012, which is 99,000 FCFA / inhabitant, the value of cattle production represents $4 \%$. This result highlights the low participation of cattle breeding in the production of food in Benin.
\end{abstract} (C) 2019 International Formulae Group. All rights reserved.

Keywords: Economic value; Livestock systems; Cattle; Animal products; Benin

\section{INTRODUCTION}

L'élevage pastoral joue un rôle prépondérant dans l'économie des pays de l'Afrique tropicale (Lesse et al., 2015). Dans l'espace ouest-africain, la demande en produits d'origine animale augmente au rythme de la croissance de la population, de l'urbanisation galopante et de l'accroissement des revenus (Sounon, 2016). Déjà en 2001, Faye annonçait que les prévisions d'évolution démographique et de croissance de la consommation individuelle de produits animaux exigent que d'ici 2020 , il va falloir produire plus de 220 milliards de litres de lait et 100 milliards de tonnes de viande dans les pays en voie de développement (Faye et Alary, 2001). Cette situation offre non seulement une garantie de marché, mais aussi des opportunités de développement du commerce régional de bétail et de lait entre le sahel qualifié de bassin de production et les pays côtiers, bassin de consommation (Sounon, 2016). Mais le paradoxe demeure la part de plus en plus croissante des importations extra africaines de produits animaux. En effet, les importations agricoles des Pays les Moins Avancés (PMA) ont enregistré une augmentation spectaculaire, passant de 2,5 milliards US\$ environ en 2000 à 32,8 milliards environ en 2015, pour représenter 2,5 pourcent du total des importations agricoles mondiales en valeur. Les exportations, en revanche, ont évolué plus lentement, atteignant tout juste 1,4 pour cent des exportations mondiales en valeur, d'où un creusement du déficit commercial des PMA sur les produits agricoles, qui s'est élevé à 15 milliards US\$ en 2015 (FAO, 2018). Dans les pays de l'UEMOA, le déficit de la balance commerciale se creuserait sous l'effet de la hausse des importations $(+1,0 \%)$, conjuguée à une baisse des exportations (-2,3\%) (UEMOA, 2016). Au Bénin, les importations de produits animaux sont évaluées en 2010 à 141.178.000 US\$ pour la viande et 
20.110.000 US\$ pour le lait (Jan et al., 2013). L'économie pastorale éprouve alors des difficultés réelles à faire face à l'accroissement de la demande potentielle de viande (de $31 \%$ dans les années 80 à $60 \%$ en 2020 ) et de lait (de $25 \%$ dans les années 80 à $52 \%$ en 2020) dans les pays en développement (Abdrahmane, 2006). L'un des défis majeurs auxquels la filière bétail /viande et lait est confronté est d'assurer aux populations un niveau suffisant de consommation de viande et de lait tout en développant les exportations (FAO et CEDEAO, 2017). Il est attendu des politiques de développement de la filière bétail/viande et lait d'influencer l'environnement économique globale de la production animale mais également la commercialisation et la consommation des produits alimentaires ainsi que les échanges sur les marchés extérieurs. Historiquement, l'élevage bovin a joué un rôle majeur dans le développement économique des pays de l'Afrique de l'Ouest, à travers sa contribution à la sécurité alimentaire et aux échanges commerciaux, en favorisant l'essor du marché local des animaux et des produits d'origine animale (Nugteren et Le Côme, 2016). De ce point de vue, la valeur économique de l'élevage bovin paraît bidimensionnelle et rend compte d'une part de sa valeur directe, qui est mesurable et quantifiable à partir de l'évaluation des échanges monétaires que génèrent ses productions, et d'autre part de sa valeur indirecte mesurable à partir des emplois qu'il pourvoit. Ainsi, aux valeurs marchandes que génère la production d'aliments que sont le lait et la viande, s'ajoute la fourniture de nombreux services dont la fumure des champs, la traction animale, la fourniture d'emplois. Pour mieux comprendre ce rôle de l'élevage bovin dans le développement économique des pays de l'Afrique de l'Ouest, une étude sur la valeur économique des systèmes d'élevage pastoraux est nécessaire. Une étude sur la valeur économique totale du pastoralisme engagée depuis 2006 par la Revue Economique Mondiale du Pastoralisme a illustré l'extrême état de pauvreté du savoir sur les économies pastorales, le manque de collecte de données dans certains pays et l'absence de désagrégation des données nationales dans d'autres pays (Luis Rodriguez, 2008). C'est pour contribuer à combler le déficit de savoirs sur les économies pastorales que la présente étude a été consacrée à l'évaluation de la valeur économique de l'élevage bovin au Bénin à partir d'un bassin de production. Cette étude s'est penchée uniquement sur la valeur économique directe des produits de l'élevage bovins (bétail, viande et lait). Elle a pour objectif de fournir des chiffres capables de générer des critères mieux avisés pour permettre l'investissement public et la prise de décisions politiques en faveur de l'élevage bovin.

\section{MATERIEL ET METHODES}

L'étude sur la valeur économique directe de l'élevage bovin s'est appuyée sur une typologie des exploitations d'élevage qui distingue deux systèmes pastoraux naisseurs: le système semi-sédentaire (SED) et le système mobile (MOB) (Sounon et al., 2015). Le milieu d'étude est un bassin de production situé à $9^{\circ} 42^{\prime} 18^{\prime \prime} \mathrm{N}$ et $1^{\circ} 40^{\prime} 11^{\prime \prime} \mathrm{E}$ (Biaou, 2006). Le bassin de la Donga, localisé dans la zone agro-écologique subhumide de l'Afrique de l'ouest, a subi ces dernières décennies des changements profonds au niveau social et de l'occupation des sols entraînant une diminution de la disponibilité en ressources pastorales et une augmentation des besoins en produits animaux (Blein et al., 2008 ; Séguis et al., 2004 ; Jouve, 2007). Son climat est unimodal, avec une pluviométrie annuelle moyenne de $1250 \mathrm{~mm}$ (Blanchard et al., 2007). Sa végétation est dominée par les savanes boisées, arborées et arbustives dont 37182 ha constituent des forêts classées (Leroux, 2012). L'accroissement annuel de la population dans le bassin est de 3,48\% depuis 1979 (INSAE, 2013). Il suit la tendance ouestafricaine qui est en moyenne de 2,6\% par an (Blein et al., 2008). Pour l'évaluation proprement dite, la démarche méthodologique suivie a comporté trois étapes: la revue et l'analyse documentaires, l'estimation des productions et leur conversion en valeur monétaire. 
La phase de revue et d'analyse documentaires a permis de visiter plusieurs sources documentaires sur les opportunités et les contraintes des productions animales, les données sur la production et la commercialisation de produits animaux en Afrique de l'ouest et au Bénin.

Les productions totales fournie par chaque système d'élevage est estimée en multipliant leur productivité moyenne par les effectifs de leur cheptel (Ba et al., 2011).

\section{Production $=$ productivité moyenne * effectifs de cheptel}

Pour estimer la productivité moyenne des systèmes d'élevage, en raison de la dispersion et de la mobilité des troupeaux, et de l'absence d'enregistrement des données démographiques animales par les éleveurs, la méthode choisie est celle rétrospective "12MO" (Lesnoff et al., 2010). Ainsi, entre février et mars 2012, pendant trois semaines, des enquêtes ponctuelles "12MO", fondées sur la mémoire des éleveurs, ont permis de reconstituer les évènements démographiques qui se sont produits les 12 mois ayant précédé l'enquête. Ainsi, des enquêtes ont été menées au niveau de 52 exploitations d'élevages bovin des deux systèmes, choisis de façon raisonnée, en tenant compte des modalités telles que: le statut foncier de l'éleveur, la taille du troupeau, le nombre de propriétaires des animaux du troupeau, la pratique de la transhumance. Les exploitations d'élevage enquêtées sont sélectionnées dans 12 localités choisies également de façon raisonnée, sur la base des critères comme : la taille des unités administratives, leur accessibilité, la taille de la population (totale, agricole, active), l'accès aux services communautaires de base (eau, école et santé), la présence d'élevages (bovins et petits ruminants). L'effectif total d'animaux du bassin utilisé est tiré des statistiques du Ministère de l'Agriculture, de l'Elevage et de la Pêche et de la FAO (FAO, 2010). Les productions moyennes d'animaux, de viande et de lait des deux systèmes d'élevage (semisédentaire et mobile) ont été estimées à l'aide d'un modèle démographique dénommé DYNMOD (Lesnoff, 2012). Les paramètres d'entrée du modèle sont la taille totale du cheptel, la structure par sexe et par classe d'âge, et les paramètres démographiques (taux de mise bas, taux de mortalité et taux d'exploitation par catégorie d'âge) qui sont estimés par la méthode 12MO. Les paramètres tels que les poids moyens des catégories d'âge, le rendement carcasse à l'abattage, le rendement lait, la durée de lactation, la proportion de femelles en lactation sont fournis par la littérature. Les valeurs utilisées pour ces paramètres sont les suivantes: le rendement moyen de la carcasse est de $47 \%$; les poids moyens des animaux sont de $53 \mathrm{~kg}$ pour les juvéniles, $166 \mathrm{~kg}$ pour les subadultes, $194 \mathrm{~kg}$ pour les vaches et $246 \mathrm{~kg}$ pour les taureaux (Youssao et al., 2000). La proportion de femelles en lactation est de $65 \%$ de l'effectif total de femelles; le rendement lait est de 2001/an/vache (MAEP, 2012; Soulé et al., 2017).

Les prix du marché utilisés pour quantifier la valeur économique directe des produits d'élevage sont pris au niveau national. Ainsi, Le prix à la ferme du lait cru est de 200 FCFA/litre (Mama Sambo, 2013), le prix moyen d'un bovin dans le bassin de la Donga est de 200000 FCFA et le Prix du Kg de viande bovine est de 1500 FCFA (MAEP, 2012). Il existe également une différence entre les prix pratiqués par les différents types d'opérateurs (éleveurs, commerçants locaux, bouchers, commerçants exportateurs) (Kadekoy-Tigague, 2002). Cette différence de prix n'est pas prise en compte.

Les formules de calcul des valeurs économiques des produits sont les suivantes :

Valeur d'animaux sur pied = productions

d'animaux * prix moyen animal ;

Valeur de la viande = productions de viande

* Prix du Kg de viande bovine ;

Valeur du lait $=$ production de lait $*$ prix à la ferme du lait cru.

\section{RESULTATS}

Les analyses nous révèlent trois résultats majeurs: (a) les paramètres démographiques des deux principaux systèmes d'élevage, (b) les quantités de 
productions (numériques, de viande et de lait) et (c) les valeurs économiques des produits.

\section{Paramètres démographiques}

Le diagnostic sur les systèmes d'élevage semi-sédentaire et mobile a ressorti des différences entre les paramètres des deux systèmes d'élevage, lesquelles, si elles sont maintenues dans le temps, pourraient engendrer des déficits de production bovine.

\section{Effectifs des cheptels}

L'estimation des effectifs des cheptels bovin des deux systèmes d'élevage du bassin en 2010, issu des statistiques officielles du Ministère de l'Agriculture, de l'Elevage, attribue 21217 têtes (53\%) au système semisédentaire et 18619 (47\%) têtes au système mobile (Tableau 1).

\section{Paramètres démographiques}

Deux catégories de paramètres démographiques sont estimées: les paramètres d'état (structures par sexe et par âge) et les taux naturels (taux de mise bas et de mortalité) (Tableau 2). Du point de vue de la répartition par sexe, pendant que le système mobile contient environ $70 \%$ de femelles, on n'en relève que $60 \%$ chez les semi sédentaires. Cette différence de proportions de femelles et particulièrement des vaches qui est de $36 \%$ chez les mobiles et de $34 \%$ chez les semi sédentaires, présage de ce que le système mobile pourrait être plus productif que celui semi sédentaire. Par contre, une comparaison des proportions par classes d'âge, ressort que la part de males subadultes de $27 \%$ est exceptionnellement élevée par rapport à la part de femelles subadultes qui est de $18 \%$, dans le système semi sédentaire. Cette situation est à l'opposé de ce qui s'observe dans le système mobile où la proportion de males subadultes $(18 \%)$ est inférieure à la proportion de femelles subadultes (25\%). Toutefois, il faut souligner que la part de mâles adultes est très faible dans les deux systèmes, $6 \%$ dans le système semi-sédentaire et $3 \%$ dans le système mobile.

Les taux de mise bas estimés dans l'analyse $12 \mathrm{MO}$, montrent que les élevages mobiles ont un taux plus élevé (0,46/vache/an) que les élevages semi sédentaires (0,39/vache/an). Cette différence se confirme par le test de comparaison qui a révélé une pvalue de 0,0173 .

D'une façon générale, les taux de mortalité naturelle (qui ne prend pas en compte les abatages) sont modérés dans les deux systèmes (inférieur à $0,10 / \mathrm{animal} / \mathrm{an}$ ). Cependant, on note quelques particularités. Chez les mobiles par exemple, la mortalité des juvéniles (moins d'un an) est la plus faible $(0,03 / a n i m a l / a n)$ alors qu'elle est la plus élevée chez les semi sédentaires (0,10/animal/an). Aussi, chez les semi sédentaires, le taux de mortalité des sub adultes est faible $(0,05 / \mathrm{animal} / \mathrm{an})$, ce qui pourrait s'expliquer par un effectif d'achat de sub adultes élevé ; ce qui atténue l'ampleur de la mortalité. Le test de comparaison des taux de mortalité entre les systèmes semi sédentaire et mobile n'est pas significatif chez les adultes. Une différence significative est notée entre les taux de mortalité chez les semi sédentaire et chez les mobiles au niveau des juvéniles.

\section{Productions des systèmes semi-sédentaire et mobile}

Les productions des systèmes d'élevages semi-sédentaire et mobile sont exprimées en nombre de têtes de bétail, en poids de viande et en volume de lait (Tableau 3). L'estimation de la production numérique révèle une production globale de 3238 têtes. La contribution du système semi sédentaire est estimée à 1318 têtes, soit $41 \%$ de la production globale; alors que celle du système mobile est estimée à 1920 têtes, ce qui représente $59 \%$ de la production globale. Pourtant, le système semi-sédentaire représente 53\% du cheptel total, contre $47 \%$ pour le système mobile. La production en viande quant à elle, est de 381 tonnes. 52\% de cette production (197 tonnes) sont fournis par le système semi-sédentaire et $48 \%$ (184 tonnes) par le système mobile. Le système semi-sédentaire qui présente une faible production numérique a contribué plus que le système mobile à cause de l'embouche qu'elle pratique. Pour le troisième produit qu'est le lait, la quantité produite est estimée à 1809000 litres. Le système semi-sédentaire en a fourni 
938000 litres $(52 \%)$ et le système mobile a fourni 871000 litres (48\%).

Valeurs économiques des productions des systèmes semi-sédentaire et mobile

La conversion monétaire des productions bovines expriment les valeurs économiques directes de la production du bassin. Ces valeurs sont respectivement de six cent quarante-sept millions six cent mille (647 600 000) francs CFA pour le bétail, cinq cent soixante-onze millions cinq cent mille (571 500 000) francs CFA en équivalent viande et trois cent soixante-un millions huit cent mille (361 800 000) francs CFA pour le lait (Tableau 4). De façon globale, la valeur économique de la production bovine du bassin est la somme des valeurs en bétail et en lait, soit un milliard neuf millions quatre cent mille (1 009400000$)$ francs CFA. Les contributions des deux principaux systèmes naisseurs sont de quatre cent cinquante-un millions deux cent mille (451 200 000) francs CFA pour le système semi-sédentaire et cinq cent cinquante-huit millions deux cent mille (558 200 000) francs CFA pour le système mobile.

Tableau 1: Effectifs des cheptels des systèmes d'élevages bovins dans le bassin.

\begin{tabular}{lccc}
\hline & \multicolumn{3}{c}{ Cheptel } \\
\cline { 2 - 4 } Systèmes d'élevage & Global & Semi-sédentaire & Mobile \\
\hline Effectifs (nb de têtes) & 39836 & 21217 & 18619 \\
\hline Proportions (\%) & 100 & 53 & 47 \\
\hline
\end{tabular}

Tableau 2: Paramètres démographiques des principaux systèmes d'élevage.

\begin{tabular}{|c|c|c|c|c|c|}
\hline \multicolumn{4}{|c|}{ Structure sexe-âge (\%) } & valeur-P & Signification \\
\hline Sexe $^{(a)}$ & Catégorie âge $^{(\mathrm{b})}$ & Semi-sédentaire & Mobile & 0,0948 & - \\
\hline $\mathbf{F}$ & JUV & 07 & 09 & 0,0033 & $*$ \\
\hline & SAD & 19 & 25 & 0,3650 & - \\
\hline & ADU & 34 & 36 & 0,1185 & - \\
\hline & TOTAL & 59 & 70 & $8,798 \mathrm{e}-06$ & $* * *$ \\
\hline $\mathbf{M}$ & JUV & 07 & 10 & $3,865 \mathrm{e}-05$ & $* * *$ \\
\hline & SAD & 27 & 18 & 0,0033 & $*$ \\
\hline & $\mathrm{ADU}$ & 06 & 03 & $6,314 \mathrm{e}-06$ & $* * *$ \\
\hline & TOTAL & 41 & 30 & 0,0948 & - \\
\hline \multicolumn{6}{|c|}{ Taux de mise bas (/femelle-année * 100) } \\
\hline & & 39 & 46 & & \\
\hline \multicolumn{6}{|c|}{ Taux de mortalité (/animal-année * 100) } \\
\hline & JUV & 10 & 03 & 0,0396 & $*$ \\
\hline & SAD & 05 & 08 & 0,1084 & - \\
\hline & $\mathrm{ADU}$ & 09 & 08 & 0,6612 & - \\
\hline
\end{tabular}


Tableau 3: Productions des systèmes semi sédentaire et mobile.

\begin{tabular}{lcccccccccc}
\hline & \multicolumn{3}{c}{ Production numérique (têtes) } & \multicolumn{2}{c}{ Production de viande (tonnes) } & \multicolumn{2}{c}{ Production de lait (1000 litres) } \\
\hline Systèmes & $\begin{array}{c}\text { Semi- } \\
\text { sédentaire }\end{array}$ & Mobile & total & $\begin{array}{c}\text { Semi- } \\
\text { sédentaire }\end{array}$ & Mobile & total & $\begin{array}{c}\text { Semi- } \\
\text { sédentaire }\end{array}$ & Mobile & total \\
\hline Quantités & 1318 & 1920 & 3238 & 197 & 184 & 381 & 938 & 871 & 1809 \\
\hline Proportions (\%) & 41 & 59 & 100 & 52 & 48 & 100 & 52 & 48 & 100 \\
\hline
\end{tabular}

Tableau 4:Valauers économiques des productions des systèmes semi-sédentaire et mobile (millions FCFA).

\begin{tabular}{lccccccccc}
\hline & \multicolumn{2}{c}{$\begin{array}{c}\text { Valeur de la production } \\
\text { numérique }\end{array}$} & \multicolumn{2}{c}{ Valeur de la production de viande } & \multicolumn{3}{c}{ Valeur de la production de lait } \\
\hline Systèmes & $\begin{array}{c}\text { Semi- } \\
\text { sédentaire }\end{array}$ & Mobile & total & $\begin{array}{c}\text { Semi- } \\
\text { sédentaire }\end{array}$ & Mobile & total & $\begin{array}{c}\text { Semi- } \\
\text { sédentaire }\end{array}$ & Mobile & total \\
\hline Montants & 263.6 & 384 & 647.6 & 295.5 & 276 & 571.5 & 187.6 & 174.2 & 361.8 \\
\hline
\end{tabular}

\section{DISCUSSION}

L'analyse de la différence de structures entre les systèmes semi-sédentaire et mobile révèle bien que la proportion très élevée de jeunes mâles dans le système semi-sédentaire ne provient pas des naissances. Il y a donc une importation de jeunes mâles qui pourrait provenir du système mobile (stratégie de complémentarité des systèmes). Autrement dit, une partie de la production du système mobile est transférée au système semi sédentaire en âge sub-adulte pour l'engraissement. C'est ce qui permet au système semi-sédentaire d'augmenter sa production en viande et dépasser celle du système mobile dont la production numérique est pourtant supérieure de 602 têtes à celle du système semi-sédentaire.

Dans cette étude, la valeur économique directe de la production bovine est représentée par l'ensemble des produits de l'élevage bovin qui sont directement vendables sur les marchés et exprimés en termes monétaires. Il s'agit principalement du bétail sur pied, de la viande et du lait. Les produits non commercialisables directement sur les marchés par l'éleveur (fumier, force de travail, laine, emploies) ne sont pas pris en compte. La traction animale est certes une réalité dans le milieu d'étude, mais la force de travail n'est pas vendue directement par l'éleveur. Les agriculteurs achètent généralement auprès des éleveurs leurs animaux de trait qu'ils gardent eux-mêmes (Houehanou et al., 2008). La situation diagnostiquée en 2012 dans le bassin de la Donga montre que les systèmes d'élevage bovin du bassin génèrent chaque année, une production totale directe équivalente à un milliard neuf millions quatre cent mille (1 009400000 ) francs CFA, soit six cent quarante-sept millions six cent mille (647 600 000) francs CFA pour le bétail et trois cent soixante-un millions huit cent mille (361 800 000) francs CFA pour le lait. La valeur économique de la viande n'est pas comptabilisée; car Le chiffre d'affaire des éleveurs repose essentiellement sur la vente des animaux sur pieds et celle du lait (Maman et al., 2018). Ce résultat indique que les éleveurs de la Donga pratiquent un élève peu spécialisé, c'est-à-dire que les objectifs sont aussi bien la production du bétail/viande que celle du lait. En effet, si la vente du bétail procure au chef de ménage les ressources nécessaires pour satisfaire les besoins des membres du ménage, l'équité dans la distribution des richesses au sein du ménage octroi aux femmes le revenu issu de la vente du lait (Chabi Toko et al., 2015). Les résultats obtenus mettent également en évidence l'importance qu'il y a à évaluer les potentialités économiques des systèmes d'élevages. Pour un marché local de 243615 
habitants, des incertitudes existent quant à la capacité de l'élevage bovin à sortir les éleveurs de la pauvreté. La valeur moyenne de production bovine est de $4143 \mathrm{FCFA} /$ habitant, alors que la FAO indique une valeur moyenne de production totale d'aliments (toutes filières confondues) de 198 I\$/habitant au Bénin en 2012 (FAO, 2013), soit 99000 FCFA/habitant (pour un taux de conversion moyen de 500 FCFA/I\$). Ce qui donne une contribution de l'élevage bovine de $4 \%$ sur la valeur totale des aliments produits par habitant. C'est la preuve que l'élevage bovin est marginalisé dans le développement économique du Bénin. Pourtant, quand on s'en tient à quelques études sur le rôle de l'élevage dans la formation des revenus des petits paysans, la conclusion est sans équivoque : c'est une source importante de revenus monétaires qui contribuent un tant soit peu à relever le niveau de vie des populations rurales (Sibiri Zoundi et Hitimana, 2008). Des études de cas en Afrique subsaharienne montrent que l'élevage apporte $34 \%$ des revenus monétaires des ménages ruraux contre $14 \%$ pour les produits végétaux et la contribution de l'élevage au PIB agricole varie de $5 \%$ en Côte d'Ivoire à $44 \%$ au Mali et fournit de l'emploi à environ $50 \%$ de la population économiquement active (Adow, 2013). L'élevage bovin a un potentiel confirmé de contribuer à chacun des huit OMD et les investissements dans le secteur de l'élevage peuvent favoriser la croissance économique de façon significative, la réduction de la pauvreté et la réalisation des OMD en Afrique (Banque Mondiale, 2014). Cependant, une transformation de l'agroéconomie autour de la production de viande et de lait est nécessaire. Un diagnostic des pertes potentielles de performances et des facteurs de risques et de vulnérabilité faciliterait l'examen de stratégies d'optimisation des processus de production pastorale et des possibilités de couverture des risques (Abdrahmane, 2006).

\section{Conclusion}

La présente étude sur la valeur économique directe de l'élevage bovin dans le bassin de la Donga s'est focalisée sur les revenus générés par les productions de bétail sur pied, de viande et du lait. Les résultats de cette évaluation révèlent que ces productions mobilisent aussi bien les performances zootechniques des animaux que les opportunités de marché. La contribution de l'élevage bovin est faible dans le développement économique du Bénin. L'étude a mis en évidence des incertitudes quant à la capacité de l'élevage bovin à sortir les éleveurs de la pauvreté, alors qu'il constitue une source importante de revenus monétaires pour relever le niveau de vie des populations rurales. Pour exploiter réellement le potentiel économique de l'élevage bovin, des investissements ciblés sont nécessaires dans le secteur afin de tirer de la production animale les ressources nécessaires à une croissance économique réelle et à la lutte contre la pauvreté.

\section{CONFLIT D'INTERETS}

Les auteurs déclarent qu'ils n'ont aucun conflit d'intérêts pour cet article.

\section{CONTRIBUTIONS DES AUTEURS}

ASKLS a participé à la conception de l'étude, à la production et à l'analyse données, ainsi qu'à la rédaction du manuscrit. EA est intervenu dans la relecture et la correction du manuscrit. LP est intervenu dans la relecture et la correction du manuscrit. AI a encadré scientifiquement l'étude depuis sa conception jusqu'à la rédaction du manuscrit. ML a conçu et mis à la disposition de l'équipe, les outils de collecte et d'analyse des données; il a lu et corrigé les résultats des analyses. SM est co-concepteur des outils de collecte et d'analyse des données. A ce titre, il a suivi et facilité la collecte des données et les analyses statistiques. $\mathrm{MH}$ a suivi et encadré scientifiquement l'étude; il a lu et corrigé le manuscrit.

\section{REMERCIEMENTS}

Nos remerciements vont à l'endroit du Centre de Coopération Internationale en Recherche Agronomique pour le 
Développement (CIRAD) qui nous a aidé à réaliser des analyses pertinentes. L'argent, c'est le nerf de la guerre... C'est pourquoi nous exprimons notre gratitude au projet "Elevage, Climat et Société" (ECLIS) qui a financé en partie la collecte de données. Merci surtout à tous les éleveurs, à toutes les autorités de la commune de Djougou, qui ont accepté de contribuer à la réalisation de cette étude et de se rendre disponibles pour les enquêtes.

\section{REFERENCES}

Abdrahmane W. 2006. Economie du pastoralisme: une analyse bibliographique. IMPD (Initiative Mondiale pour un Pastoralisme Durable) - IUCN EARO: Dakar, Sénégal, 23 p.

Adow SS. 2013. La place de l'élevage pastoral dans l'économie et les politiques nationales et régionales. In la contribution de l'élevage pastoral à la sécurité et au développement des espaces saharo-sahéliens, N'Djamena, 27-29 MAI 2013. N'Djamena, Tchad, 8 p.

Ba A, Lesnoff M, Coulibaly D, Chapuispoccard R, Moulin C-H. 2012. Un outil simple de projection démographique pour estimer la productivité d'un cheptel: application à un cheptel bovin du Mali sud. Dans Partenariat, Modélisation, Expérimentation: Quelles Leçons Pour La Conception de l'innovation et l'intensification Écologique, BoboDioulasso, novembre 2011. Cirad: BoboDioulasso, Burkina Faso, 8 p.

Banque Mondiale. 2014. Investir dans le secteur de l'élevage L'importance de chiffres précis. Banque Mondiale - FAO: Washington, USA, $154 \mathrm{p}$.

Biaou CF. 2006. Monographie de la commune de Djougou. AFRIQUE CONSEIL: Cotonou, Bénin, 48 p.

Blanchard M, Peugeot C, Seghieri J. 2007. Structure et dynamique saisonnière de la végétation sur quatre types de couvert végétal représentatif de la diversité du bassin versant de la Donga. Biogeosci., 65194: 2193-2205.
Blein R, Soulé BG, Dupaigre BF, Yérima B. 2008. Les potentialités agricoles de l'Afrique de l'Ouest (CEDEAO). FARM) $116 \mathrm{p}$.

Chabi Toko R, Adégbidi A, Lebailly P. 2015. Valorisation des produits laitiers dans les ménages Peul du Nord-Est du Bénin. Int. J. Biol. Chem. Sci., 9(6): 2716-2726. DOI: http://dx.doi.org/10.4314/ijbcs.v9i6.16

CIRAD. 2008. 12MO : une méthode rétrospective pour l'estimation des paramètres démographiques des cheptels de ruminants domestiques. CIRAD: Montpellier, France, 128 p.

FAO. 2010. FAOSTAT.data. FAO: Rome, Italie.

FAO. 2013. Food security indicators. FAO: Rome, Italie.

FAO. 2018. La situation des marchés des produits agricoles 2018. Commerce agricole, changement climatique et sécurité alimentaire. FAO: Rome, Italie, $119 \mathrm{p}$.

FAO, CEDEAO. 2017. Revue des filières bétail/viande et lait et des politiques qui les influencent au Niger. FAOCEDEAO: Rome, Italie, $122 \mathrm{p}$.

Faye B, Alary V. 2001. Les enjeux des productions animales dans les pays du Sud. INRA Prod. Anim., 14(1): 3-13.

Houehanou T, Houinato M, Adandédjan C, Gbangboché AB, Hounzangbé-Adoté MS, Sinsin BA. 2008. Gestion pastorale et structure des terroirs agricoles dans la périphérie de la Djona (Nord-Est Bénin). Int. J. Biol. Chem. Sci., 2(4): 497-507.

INSAE. 2013. Résultats provisoires du quatrième recensement général de la population et de l'habitation. INSAE

Jan VDL, Hans S, Roel B, Evelien DO, Sifra B, Jessica C. 2013. Aid and Trade for Livestock Development and Food Security in West Africa. Wageningen UR Livestock Research: Wageningen, $745 p$. http://www.livestockresearch.wur.nl

Jouve P. 2007. Le jeu croisé des dynamiques agraires et foncières en Afrique 
subsaharienne. Cah. Agric., 16(5): 379385.

Kadekoy-Tigague D. 2002. Rentabilité et dynamique du commerce de bovins sur pied en République. Cirad: Montpellier, France - Prasac: N'Djamena, Tchad, 7 p. https://hal.archives-ouvertes.fr/hal00131033

Leroux L. 2012. Analyse diachronique de la dynamique paysagère sur le bassin supérieur de l'Ouémé (Bénin) à partir de l'imagerie Landsat et MODIS- Cas d'étude du communal de Djougou. ANR ESCAPE - Hydrosciences Montpellier: Montpellier, 62p.

Lesnoff M. 2012. Basedemog - A simple database to gather demographic parameters of tropical livestock herds. Cirad: Montpellier, France, 14p. http://livtools.cirad.fr

Lesse P, Marcel H, Jonas D, Hippolyte D, Bouraima Y, Ismael T, Brice T, Brice S. 2015. Transhumance en République du Bénin : états des lieux et contraintes. Int. J. Biol. Chem. Sci., 9(5): 2668-2681. DOI:

http://dx.doi.org/10.4314/ijbcs.v9i5.37

MAEP. 2012. Annuaire statistique sur l'élevage. MAEP: Cotonou, Bénin, 79 p.

Mama Sambo A. 2013. Etude relative à la formulation $\mathrm{du}$ programme d'actions détaillé du développement de la filière lait en zone UEMOA. Cirad: Montpellier, France, 44 p.

Maman LAA, Chaibou M, Mani M, Garba MM, Gouro AS. 2018. Pratiques d'éleveurs et résultats économiques d'élevage dans les exploitations urbaines et périurbaines de Niamey. Int. J. Biol. Chem. Sci., 12(1): 294-309. DOI: https://dx.doi.org/10.4314/ijbcs.v12i1.24

Nugteren H, Le Côme C. 2016. Libérer le potentiel du pastoralisme pour développer l'Afrique de l'Ouest. KIT: Pays-Bas - Banque Mondiale: ÉtatsUnis, 94 p.

Rodriguez L. 2008. Une perspective mondiale sur la valeur économique totale du pastoralisme: Un rapport de synthèse global basé sur six évaluations pays. Institut International de Recherche sur l'Élevage, Nairobi, 29 p.

Séguis L, Galle S, Peugeot C, Kamagate B, Robain H. 2004. Caractérisation des processus hydrologiques sur le super-site de la Donga. Haut bassin de l'Ouémé (Bénin). Dans Atelier Expérimentation et Instrumentation, Paris, France, 23-24 mars 2004. INSU - METEO France IFREMER: Paris, 6 p.

Sibiri ZJ, Hitimana L. 2008. Elevage et marché régional au Sahel et en Afrique de l'Ouest: Potentialités et défis. CEDEAO - CSAO/OCDE: Paris, France, 182 p.

Soulé HA, Djenontin AJP, Kpéra GN, Mensah GA. 2017. Production laitière bovine au Nord-Bénin. Bulletin de la Recherche Agronomique du Bénin (BRAB), Numéro 81: 18-25.

Sounon A. 2016. Diversité, vulnérabilité et adaptation de l'élevage bovin dans le bassin de la Donga au Bénin: changement du milieu et transformation des systèmes. Thèse Doct., Université d'Abomey-Calavi: Abomey-Calavi, Bénin, 257p.

Sounon A, Ickowicz A, Lesnoff M, Houinato M. 2015. Typologie des systèmes d'élevage bovin en vue d'une caractérisation des rapports entre l'élevage et son environnement dans le bassin de la Donga au Bénin. Dans Actes Du 4ème Colloque Des Sciences, Cultures et Technologies de l'UAC Bénin, (Conseil Scientifique de l'UAC), Abomey-Calavi, Bénin, 23-28 septembre 2013. Université d'Abomey-Calavi, Abomey-Calavi, Bénin, p. 97-117.

UEMOA. 2016. Rapport annuel 2016 sur le fonctionnement et l'évolution de l'union. UEMOA, 94 p.

Youssao AKI, Ahissou A, Toure Z, Leroy PL. 2000. Productivité de la race Borgou à la ferme d'élevage de l'Okpara au Bénin. Rev. Elev. Méd. Vét. Pays Trop., 53(1): 67-74. 